\title{
4.2 Eintrag zu „Paracelsus” im Biographischen Lexikon der hervorragenden Aerzte aller Zeiten und Völker
}

482

\author{
PAPPENHEIMI. - PARACELSUS.
}

den Comptes rendus (von 1846 an) und erbielt 1847 den grossen Preis fur eine von der Acad. des sc. ausgeschriebene Preisaufgabe, betreffend die Zeugungsorgane der 5 Classen von Wirbelthieren. Später gerieth er mit Flodrens in Misshelligkeiten, verliess Paris im Groll und begab sich 1849 - er hatte damals sein nicht unbetrllichtliches vaterliches Erbtheil ausbezahlt erhalten - auf eine wissenschaftlicho Forschungsreise nach Amerika, wo er volle 10 Jahre ganz verschollen war. Angeblich hatte er behufs sprachvergleich. Studien eine ganze Reihe eingeborener Indianerstamme besucht. In der Havanna am gelben Fieber erkrankt, wurde er fast sterbend von Deutschen aufgefunden, geheilt und 1859 nach Hamburg und Breslau zurllokspedirt. 1861 siedelte er nach Berlin uber, war daselbst dem Namen nach prakt. Arzt, lebte aber als Sonderling, anscheinend in den armlichsten. Verhaltnissen, obgleich von seinen Verwandten unterstutzt, war ein ständiger Gast der Bibliotheken, wo er allerlei med. und Sprachstudien trieb und starb in einem Krankenhause 10. Febr. 1882. Leopoldina, 1882, pag. 48, 122. - Callisen, XXXI, pag. 142. - Catalogue of Scientifle Papers. IV, pag. 753.

Pappenheim, Lou is P., geb. 25. Nov. 1818 zu Oppeln, studirte und promovirte 1841 in Berlin mit der Diss. "De cellularum sanguinis indole ac vita. Observationes microscopico-chemicae", war Kreis-Physicus in Kosten (Prov. Posen), wurde Privatdocent (1858) in Berlin und 1860 Regierungs- und Medicinalrath zu Arnsberg in Westfalen, Toselbst er im Mai 1875 verstarb; er ist besonders bekannt durch sein grosses "Handbuch der Sanitätspolizei. Nach eigenen Untersuchungen bearbeitet" (2 Bde., Berlin 1858-59; 3. Suppl.-Bd. Ib. 1864; 2. Aufl. Ib. 1868-70). Ausserdem gab er heraus: "Monatsschrift für exacte Forschung auf dem Gebiete der Sanitätspolizei" (Ib. 1859-60) - „Beitrüge zur exacten Forschung auf dem Gebiete der Sanitätspolizei" (Ib. 1860-62).

Dechambre, 2. Série, $\mathrm{xx}$, pag. 499.

Pgl.

Paracelsus, Philippus Aureolus Theophrastus Bombastus P. ab Hohenbein, auch "Helvetius Eremita" genannt, war 1491 in einem Hause nahe an der uber die Sihl fuhrenden Teufelsbrlleke, eine Stunde weit von dem berilhmten Wallfahrtsorte Mraria Einsiedeln (Canton Schwyz), geboren. Sein Vater, der Arzt $\pi^{r} i l h e l m$ ab Hohenheim, stammte aus der alten und berulhmten schwabischen Adelsfamilie der Bombaste, die sich von dem adeligen Schloss Hohenheim, dem nachmaligen Esslinger Hof, bei dem Dorfe Pfunningen bei Stuttgart "Bombaste von Hohenheim" nannten. 1502 siedelte er mit seinem Vater nach Villach in Kürnten tuber, wo letzterer 1534 starb. ${ }^{\circ}$ Er bezog 1006 die Universitat zu Basel, gab sich hier besonders chem. Studien hin, lebte lăngere Zeit bei dem als Alchemist bekannten TriTnEsurus, machte dann grössere Reisen duroh Europa, hielt sich an verschiedenen Universitäten Studien halber auf und genoss bereits um 1527 wegen seiner Kenntnisse einen solchen Ruf, dass er auf des bekannten Kirchenvaters, seines Landsmannes 0 ekol a mpadius Empfehlung als Nachfolger von COPUS 1527 zum Stadtarzt von Basel ernannt wurde. In dieser Eigenschaft trat er in ebenso abenteuerlioher, wio verletzender Weise auf, indem er als Zeichen seiner Verachtung der arabisohen Med. alle Werke des AvicENNA auf öffontlichem Markt verbrannte. Noch mehr brachte er seine Collegen gegen sich auf, als er es wagte, sich der deutschen Sprache zu bedienen. Es kam schliesslich zu völligem Zerwürniss, als er auf das schmutzige geschuftliche Treiben der im Bunde mit den Apothekern stehenden Aerzte aufmerksam und demselben ein Ende machte. Zuletzt zerfiel er auch nooh mit den Behörden; or liess aus Aerger daruber dieselben in Pamphleten angreifen, musste aber in Folge dessen heimlich aus Basel entweichen und fuhrte nun ein abenteuerliches Wanderleben, das ihn durch den Elsass, die Schweiz, Deutschland und bis an die entferntesten Punkte Europas, selbst bis nach Asien hinein verschlug, wobei er von einer grossen Zahl von Schtllera begleitet war, meistens Vagabunden, die nur den Stein der Weisen kennen lernen wollten und P. fü den Besitzer desselben hielten.

Eintrag von Julius Pagel zu „Paracelsus” im Biographischen Lexikon der hervorragenden Aerzte aller Zeiten und Völker. Hrsg. v. August Hirsch. Bd IV. Wien, Leipzig: Urban \& Schwarzenberg 1886, S. 482-485. 
Allmallig verbreitete sich sein Name und sein Ruhm ther ganz Europa. Er wurde 1540 nach Salzburg berufen, lebte aber hior nur noch $1 \mathrm{Jahr}$ und starb daselbst 24. Sept. 1541. - P. ist jedenfalls für die nystische Richtung der Med. im 16. Jahrh. die einflussreichste Persönlichkeit gerwesen. Es ist uber ihn sehr viel gedacht und geschrieben worden. Yon der einen Seite ist er als Reformator, Yater der Heilkunde, Begrunder der nenen Richtung in der Med. gepriesen und in den Himmel erhoben, von der anderen als ein Mystiker, Schwirmer, Betrliger, Trunkenbold, Gaukler bezeichnet worden. Dieser Widerspruch löst sich, wenn man auf die Quellen zurllckgeht, aus denen er sich ergeben hat. Nachgerriesenermassen ist der bei Weitem grösste Theil aller Sehriften, dic unter seinem Namen erschieven sind, untergeschoben und von den tbrigen ist es höchst fraglich, wieviel davon ihm angehört und wieviel von seinen Freunden und Feinden hineingearbeitet worden ist. Seine Freunde glaubten, seine Lebre gar nicht dunkel genug darstellen zu könuen und seine Feinde, die ihn zu verunglimpfen bestrebt waren, scheuten sich nicht, in seine. Schriften einen Trust von Unsinn hineinzutragen. Selbst dio von seinen Gegnern gegen ihn erhobenen Angriffe sind darin als sein eigenes Werk aufgefubrt. - Als Quelle des Wissens bezeichuete P. die Iorfabrung, aber nicht dic auf den Wege einer rationellen inductiven Forschung gerionnene Kenntniss, sondern das aus der neueren Pbilosophie geschöpfte aprioristische Wissen; vorzugsweise ist es die Analogie, aus der er die Erkenntniss ableitet. Sein System basirt wesentlich auf den 'Theo- und Kosmosoplien der Neuplatoniker, in die er eben nur das neuere philosophische Element hineingebracht hat. Die ganze Welt wird von ihm streng pantheistisch aufgefasst. Er unterscheidet 3 Sphären: eine himmlische mit den reinen Intelligenzen und körperfreien Seelen, einc astralische Welt mit den seelenlosen Elementargeistern und eiue körperliche Welt, d. i. die irdische Natur, die an beiden genannten Sphären Theil nimmt. In dem Menschen sind nun alle 3 Sphären miteinander verbunden. Er ist der Mrikrokosnus in dem Makrokosmus. Alles Geschaffene wird auf ein Magisterium maguum zurllckgefulhrt u. s. w. Die Basis der ganzen körperlichen WVelt bilden die 4 Aristotelischen Elemente; diese finden sich, körperlich gedacht, in 3 Stoffeu wieder, dio P. von seinem chemischen Standpunkte aus sich durch ihre clsemischen Eigenschaften von einander unterscheiden lusst. Diese 3 Stoffe sind: 1) Salz, d. i. der Begriff des Festen, aller der chenischen Stoffe, die durch das Feuer unzerstörbar sind; 2) Quecksilber, d. i. der Begriff des Flussigen, das durch Feuer unver:ndert verflussigt wird; 3) Schwefel, d. i. der Begriff des Luftigen, das durch das Feter nicht bloss zerstörbar, sondern auch gleichzeitig verănderlich ist. Diese 3 Begriffe werden rein symbolisel von $P$. gebraucht fur alle Stofie, die sich ebenso in Bezug auf ihr Verlialten dem Feuer gegentlber qualificiren lassen. Das eigentliohe Wesen liegt aber nicht in dem Stofie, sondern in dem Geiste, in dem ihm eingepflanzten göttliohen Keime, in den Krüften, die sich aus ihm entfalten; diese werden als Quinta essentia, häufig auch als Archaeus, als das eigentlich Belebende von ihm bezeichnet. Der Mensch besteht nun, indem er an jenen 3 Sphären sich betheiligt, ans sicht- und fuhlbarem Körper, sodann aus einer körperlichen Seelo (Archneus), der eben dio an dem Organismus hervortretenden lebendigen Eigenschaften vermittelt und endlich aus der unsterblichen Seele. In seiner Pathologie interscheidet er 4 Gruppen von Krankheitsursachen, sog. „Entia“: 1) Ens deale, die göttliche Schickung; 2) Ens astrale, Einfluss der Gestirne, der sich namentlich als Quelle der pestilenzialischen, epidemischen Krankheiten kundgiebt; 3) Fns spirituale, archacus, das geistige Element im Nenschen, das or sich ubrigens körperlich vorstellt; endlich 4) das Ens naturale, die den ganzen Menschen umgebende körperliche Welt, a) als Ens alimentorum ot veneni und b) als Ens seminis, dis Ursache der angeborenen und heredittiren Krankheiten. Jede Krankheit kann nun vou einem dieser Entia ihren Ursprung nelmen, indem diese eine chemische Einwirkung auf den Organismus hervorbringen, auf chemischem Wege Trennungen und neue Verbindungen bilden. Salz, Schwefel und Mercur erfahren mannichfache Verånderungen. Die Art, wie P. 
uun die einzelnen Krankheitsformen auf die verschiedenen Veränderungen zurlickfubrt, ist weiter nichts als ein subtiles Spiel mit Analogieen. - - Den Mittelpunkt seiner Lebre bildet die Therapie. Der erkrankte Organismus wird gesund einerseits durch die ihm immanente Kraft, die er als Archaeus darstellt, dio qúct; der griechischen Aerzte, und ausserdem durch directe arztliche Eingriffe, und zwar nicht in den Sinne der alten Medicin Contraria contrariis, sondern es muss nach P. dieser Gegensatz zwischen Krankheit und Heilmittel in den Ideen liegen. Er sagt z. B. Massersueht ist im Mikrokosmus Das, was Ueberschwemmung im Makro. kosmus ist; so wie man nun Ueberschwemmung beseitigt durch Ableitung von Wasser, so glaubt er auch durch reichliche Entleerung die tlbermassige Feuchtigkeit zu entfernen. Die Kolik z. B. vergleicht er mit dem Winde und verlangt Wärme und Trockenheit bei der Behandlung derselben. Atrophieen und Phthisen vergleicht er mit der Austrocknung und empfiehlt die entsprechenden Mittel, die der Idee nach direct entgegengesetzt sind u. s. w. Eine zweite Methode der Behandlung ist die durch Areana, d. h. durch Substanzen, die durch die in ihnen inneriohnende magische Kraft. die in dem Organismus wuchernde Abnormität zerstören, also die Krankheiten in ihren Wurzeln angreifen. Man muss nach P. dahin kommen, die lirankheiten nicht nach den anatomisch ergriffenen Theilen, sondern nach dem entsprechenden Heilmittel zu benennen. Er unterscheidet also z. B. Morbus terebinthinus, helleborinus etc. (in der allerneuesten Zeit hat diese Lehre in RADEsacHER wicder ibren Apostel gefunden). P. giebt nun auch Vorschriften uber die Art, die Heilmittel für die einzelnen Krankheiten zu entdecken. Er lehrt, dass die Natur selbst auf sie hinweise durch die eigenthilmliche Gestaltung, Farbe etc. der Pflanzen, namentlich aber durch die Aehnlichkeit, die diese Stoffie mit gewrissen Organen des Körpers haben, die auch als Organheilmittel als solche anzusehen seien. Hieraus entwickelte sich die Lehre von den sog. "Signaturen", welche die Pflanzenstofle tragen und aus denen ein Schluss auf ihre Wirkung als Heilmittel gezogen wurde. Bei denon, die solche Signaturen nicht tragen, entscheidet lediglich das Experiment. Um nun die Wirkung dieser Arcana festzustellen, wurde es nothweudig, die Heilmittel genau zu studiren. Auf seine Veranlassung sind denn auch zahlreiche metallische Heilmittel zuerst für den innerlichen Gebrauch in die Therapic eingefubrt worden, so: Eisenoxyḍ, Mercur, Antimon, Blei, Kupfer, Arsen, Schwefelprilparate u. s. w., die bis dahin so wenig angewandt worden waren, dass, als P.'s Lebre grössere Verbreitung fand, von Seiten verschiedener Facultăten dagegen opponirt und sogar die Regierungen veranlasst wurden, ein Verbot gegen den innerlichen Gebrauch der metallischen Mittel zu erlassen. Die Paracelsische Lohre fulhrte ausserdem zu einer Darstellung der in der Pflanze vorhandenen Kräfte, da nicht die ganze Pflanze heile, sondern nur einzelne Stoffe derselben. So entstanden die Tincturen, spirituösen Extracte etc. - P. hat seine Schriften, deren Zahl sich auf 364 belaufen soll, meistens deutsch geschrieben, resp. dictirt und im Manuscript verkauft. Viele derselben sind von seinen Anhängern in's Lateinische ubersetzt und zum Druck befördert worden. Sie sind mehrfach gesammelt von J. HoSER (10 voll., Basel 1589-91; Frankfurt 1603, 3 voll., fol.; Strassb. 1605); von F. PETiscus: "Opera omnia medico-chemico-chirurgica" (3 voll., fol., Genf 1658; 11 voll., "Basel 1575; 12 voll., Frankf. 1603). Als dic wichtigsten der echt befundenen nennen wir: "De gradibus et compositionibus receptorum" (geschr. 1526) - "Die kleine "Chirurgie. Von französischen Blatern, Lähme, Beulen etc." (1528, bildet den 4. Theil der "Grossen Wundparznei") - "Siehen Bücher von allen offenen Schäden, so aus der Natur geboren werden" $(1528,3$ Thle. der "Grossen Wundarznei") - "Vom Holtz Guajaco" (1529) - "Von den Imposturen der Aerate" (1530) - „Opus paramirum ad medicam industriam" (1531) - die beiden ersten Bucher der "Grossen Wundarznei" (1536 u. 37) - "Paragranum" (1538). Die Sprache, dic P. fulhrt, ist sehr derb, nit oft plumpen Ausdr'llcken und wo es gilt, feindliche Angrific zu widerlegen, sehr auffallend und den guten Geschmack - nach 
der Sitte der damaligen Zeit - völlig bei Seite setzend. Er hatte eine gauz tilchtige wissenschaftliche Bildung, wis sich das auch in seiner Verehrung des HIPPOKRATES kundgiebt, doch war er allerdings im böchsten Grade der mystischen Richtung seiner Zeit verfallen, dabei aber vom eigentlichen Aberglauben, von allen den Thorheiten der Astrologen und Alchemisten weit entfernt, die er selbst sogar lächerlich machte, indem er sagte, dass jeder Mensch die Damonen ete. in seinem eigenen Busen trage. Im Publicum fand seine Lehre ausserordentlich vielen Anklang; dagegen hat er eigentliche Schuler nicht gehabt. Er spricht es selbst sehr oft aus, dass, wenn man Alle zusanmenzăhlt, die seine Schuler heissen wollten, Hunderte herauskämen, aher darunter waren nur $2-3$, die ibn verstanden bătten, die ulbrigen seien Lumpen, die nur von ihm die angeblichen geheimen Kilnste bătten lernen wollen. Biogr. méd. VI, pag. 361. - Dict. hist. III, pag. 665. - JI. B. Lessing, P., sein Leben und Denken. Berlin 1839 . - JI oll in Württemb. ärztl. Correspondenzbl. 1851, 33. Allgen. Deutsch. Biogr. XII, pag. 675. Pagel.

Paradys, Nicolaas P., geb. 1740 in Amsterdam, studirte in Leyden unter D. van ROYEN, F. B. Albinus und Gaubius und promovirte $1763 \mathrm{zmm}$ Dr. med. mit einer "Diss. pathologica qua inquiritur in naturam inflamma. tionis ${ }^{\prime}$. Darauf ging er nach Paris, wo er LEVRer, LoRRY und SAxcuez folgte und nach Rouen, um LE CAT zu hören. Er etablirte sich in Amsterdam, wo sein Vater, David P., auch die Praxis ausibte; doch wurde er $178 \pm$ als Stellvertreter yon weiland van Doeveres als Prof. der Med. nach Leyden gerufen ("Oratio de diligenti therapeutices universalis studio, maximo recte medendi instrumento" $) .1790$ zum Leibarzt des Prinzen Willem V. ernannt, war er auch als consultirender Arzt sehr beliebt. 1800 wurde ihm der Unterricht in der Geschichte der Medicin tbertragen ("Oratio de cognitione historiae medicin'le magno, cum ad medici in arte exercenda solertiam, tum ad artis amplificationem

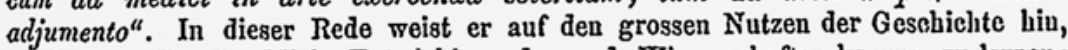
um dadurch die allmkhliche Entwicklung der med. Wissenschaften kennen zu lernen; alich bat er eine ausgezeichnete Skizze von den Verdiensten Hiprokkates' und BOERHAAVE's gegeben, so dass dieselbe noch heute eine sehr leseuswilidige Arbeit genannt werden muss. Seine Yorlesungen uber specielle Pathologie und Therapie und uber Materia medica waren immer sehr besucht, da P. ausser seiner tilchtigen Kenntniss und grossen Belesenheit, auch ein ausserordentliches oratorisches Talent hatte („qui non ninus ob praeclaram artis medioae scientiam, quam ob doctrinam cum nostratum literarum elegantia conjunctam, delectari solebat", gagt RuHNKENres) und sich um den ihm anvertrauten Unterricht ausserordentlich viel Mrilhe gab. Dass er daboi den Aphorismen von BOERHAAve folgte, kann nicht verwundern, obgleich er die darin vorkommenden Fehler jedesmal hervorhob und corrigirte. P. starb 1812. Ausser den genannten Reden haben wir von ihm noch eiue "De euthanasia naturali" (Leyden 1794), wahrend seine "Institutiones practicae " und die daran 1785 als Einleitung gefugten "Lectiones publicae" in den nach seinem Tode durch seinen Soln herausgegebenen "Opuscula academica" (Loyden 1813) publicirt sind.

G. C. B. Suringar, Gesch. van het geneesk. onderwys aan de Leydsche Hoogoschool. C. E. Daniěls.

Parat, Philibert P., geb. im Sept. 1763 zu Lyon, studirto und promovirte 1790 in Montpellier, zeichnete sich wahrend der Belagerung von Lyon aus, diente mehrere Jahre bei der Alpen-Armee als Chirurg.-major., wurdo spater, nach Lyon zurlickgekehrt, Arzt am dortigen Hôtel-Dieu und starb 11. Dec. 1838. Er schricb: „Sur les moyens de perfectionner les études de l'art de guérir" (Lyon 1791) - ${ }_{n}$ Obs. médicales sur les principaux effets du froid et du chaud sur le sommet des hautes montagnes" (zus. mit Martin jeune in Recueil des actes de la Soc. de santé de Lyon an I) - "Réflexions pratiques sur la gale" (Ib.) "Eloge historique de Marc-Antoine Petit" (Lyon 1812) u. A. m. pag. 143. Dechambre, 2. Sério, XXI, pag. 117. - Callisen, XIV, pag. 295; XXXI, 